\title{
Advance Implementation for Enhance Image using Neural Network and Optimal Image Processing Algorithm in Digital Image Processing
}

\author{
Indra Kishor ${ }^{1}$, Dhara Upadhyay ${ }^{2}$ \\ ${ }^{1,2}$ Assistant Professor, Department of computer science \& Engineering, \\ Arya Institute of Engineering and Technology. Kukas, Jaipur, Rajasthan
}

\begin{abstract}
We review more than 200 applications of neural networks in image processing and discuss the present and possible future role of neural networks, especially feed-forward neural networks, Kohonen feature maps and Hopleld neural networks. The various applications are categorized into a novel two-dimensional taxonomy for image processing al-gorithms. One dimension speciles the type of task performed by the algorithm: preprocessing, data reduction=feature extraction, segmentation, object recognition, image understanding and optimization. The other dimension captures the abstraction level of the input data processed by the algorithm: pixellevel, local feature-level, structure-level, object-level, object-set-level and scene characterization. Each of the six types of tasks poses specilc constraints to a neural-based approach. These specilc conditions are discussed in detail. A synthesis is made of unresolved problems related to the application of pattern recognition techniques in image processing and specilcally to the application of neural networks. Finally, we present an outlook into the future application of neural networks and relate them to novel developments.
\end{abstract}

Keywords: Digital image processing; Invariant pattern recognition; Preprocessing; Feature extraction; Image compression; Segmentation; Object recognition; Image understanding; Optimization

\section{Introduction}

Techniques from statistical pattern recognition have, since the revival of neural networks, obtained a widespread use in digital image processing. Initially, pattern recognition problems were often solved by linear and quadratic discriminants [1] or the (non-parametric) k-nearest neighbour classi1er and the Parzen density estimator [2,3]. In the mideighties, the PDP group [4] together with others, introduced the back-propagation learning algorithm for neural networks. This algorithm for the 1rst time made it feasible to train a non-linear neural network equipped with layers of the socalled hid-den nodes. Since then, neural networks with one or more hidden layers can, in theory, be trained to perform virtu-ally any regression or discrimination task. Moreover, no assumptions are made as with respect to the type of underlying (parametric) distribution of the input variables, which may be nominal, ordinal, real or any combination hereof.

In their 1993 review article on image segmentation, Pal and Pal predicted that neural networks would become widely applied in image processing [5]. This prediction turned out to be right. In this review article, we survey applications of neural networks developed to solve different problems in image processing (for a review of neural networks used for 1D signal processing, see Ref. [6]). There are two central questions which we will try to answer in this review article:

1) What are major applications of neural networks in image processing now and in the nearby future?

2) Which are the major strengths and weaknesses of neu-ral networks for solving image processing tasks?

To facilitate a systematic review of neural networks in image processing, we propose a two-dimensional taxonomy for image processing techniques in Section 2. This taxonomy establishes a framework in which the advantages and unresolved problems can be structured in rela-tion to the application of neural networks in image pro-cessing (Section Section 4 discusses some real-world applications of neural networks in image processing. In Section 5, identiled problems are considered and Section 6 presents an overview of future research issues which need to be resolved or investigated further as to expedite the application of neural networks in image processing. A number of future trends are also brieGy sketched.

In the paper, we will not consider the basic theory of neural networks. The reader is referred to standard text books, e.g., Ref. [7].

\section{Taxonomy for image processing algorithms}

Traditional techniques from statistical pattern recognition like the Bayesian discriminant and the Parzen windows were popular until the beginning of the 1990s. Since then, neural networks (ANNs) have increasingly been used as an alternative to classic pattern classilers and clustering techniques. Non-parametric feed-forward ANNs quickly turned out to be attractive trainable machines for featurebased segmentation and object recognition. When no gold standard is available, the self-organising feature map (SOM) is an interest-ing alternative to supervised techniques. It may learn to discriminate, e.g., diIerent textures when provided with powerful features. The current use of ANNs in image processing exceeds the aforementioned traditional applications. The role of feed-forward ANNs and SOMs has been extended to encompass also low-level image processing tasks such as noise suppression and image enhancement. Hopleld ANNs were introduced as a tool for 1nding satisfactory solutions to complex (NP-complete) optimisation problems. This makes them an interesting alternative to

\section{Volume 6 Issue 12, December 2017}




\section{International Journal of Science and Research (IJSR) \\ ISSN (Online): 2319-7064}

Index Copernicus Value (2016): 79.57 | Impact Factor (2015): 6.391

traditional optimisation algorithms for image processing tasks that can be formulated as optimisation problems.

The dilerent problems addressed in the 1eld of digi-tal image processing can be organised into what we have chosen to call the image processing chain. We make the following distinction between steps in the image process-ing chain (see Fig. 1):

1) Preprocessing=1ltering. Operations that give as a result a modiled image with the same dimensions as the original image (e.g., contrast enhancement and noise reduction).

2) Data reduction=feature extraction. Any operation that extracts signilcant components from an image (win-dow). The number of extracted features is generally smaller than the number of pixels in the input window.

3) Segmentation. Any operation that partitions an image into regions that are coherent with respect to some criterion. One example is the segregation of diIerent textures.

4) Object detection and recognition. Determining the position and, possibly, also the orientation and scale of specilc objects in an image, and classifying these objects.

5) Image understanding. Obtaining high level (semantic) knowledge of what an image shows.

6) Optimisation. Minimisation of a criterion function which may be used for, e.g., graph matching or object delineation.

Optimisation techniques are not seen as a separate step in the image processing chain but as a set of auxiliary techniques, which support the other steps.

Besides the actual task performed by an algorithm, its processing capabilities are partly determined by the abstraction level of the input data. We distinguish between the following abstraction levels:

a) Pixel level. The intensities of individual pixels are provided as input to the algorithm.

b) Local feature level. A set of derived, pixel-based fea-tures constitutes the input.

c) Structure (edge) level. The relative location of one or more perceptual features (e.g., edges, corners, junc-tions, surfaces, etc.).

d) Object level. Properties of individual objects.

e) Object set level. The mutual order and relative location of detected objects.

f) Scene characterisation. A complete description of the scene possibly including lighting conditions, context, etc.

\section{Neural networks in image processing}

In this section, we will review neural networks trained to perform one of the six tasks in the image processing chain (3.1-3.6).

3.1. Preprocessing The 1rst step in the image processing chain consists of preprocessing. Loosely de1ned, by preprocessing we understand with following diagram.

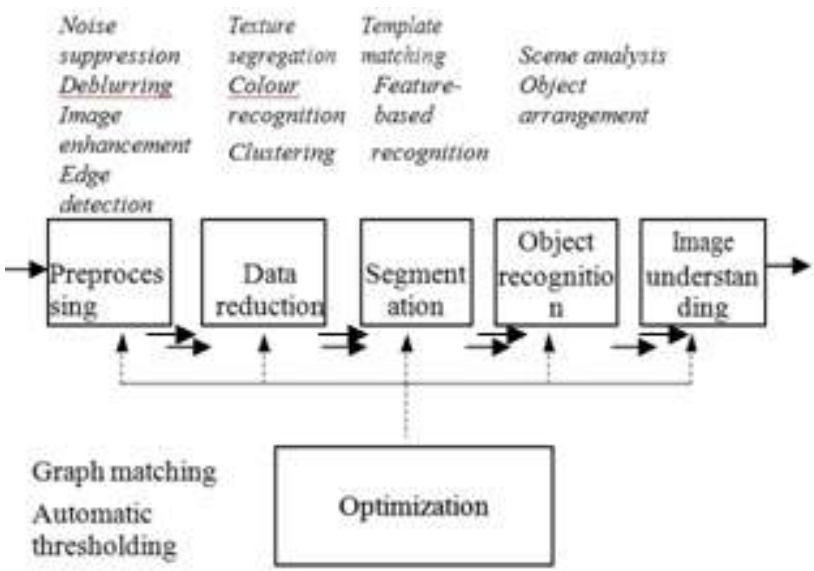

Figure 1: The image processing chain containing the 1ve deferent tasks: preprocessing, data reduction, segmentation, object recognition and image understanding. Optimization techniques are used as a set of auxiliary tools that are available in all steps of the image processing chain.

\subsection{Tables}

The image processing tasks categorized into a twodimensional taxonomy mean any operation of which the input consists of sensor data, and of which the output is a full image. Preprocess-ing operations generally fall into one of three categories: image reconstruction (to reconstruct an image from a number of sensor measurements), image restoration (to remove any aberrations introduced by the sensor, including noise) and image enhancement (accentuation of certain desired features, which may facilitate later processing steps such as segmentation or object recognition). categories will be discussed separately below. The majority of the ANNs were applied directly to pixel data (level A); only four networks were applied to more high-level data (features, level B).

Table 1 contains the taxonomy of image processing algorithms that results from combining the steps of the image processing chain with the abstraction level of the input data.

\begin{tabular}{|c|c|c|c|c|c|c|}
\hline & Preprocessing & Compression feature extract & Segmentation & Recognition & Image understanding & Optimization \\
\hline Pixel & 26 & 25 & 39 & 51 & 3 & 5 \\
\hline Feature & 4 & 2 & 19 & 38 & 2 & 3 \\
\hline Structure & & & 2 & 6 & & 5 \\
\hline Object & & & & & 2 & 1 \\
\hline Object set & & & & & & \\
\hline Scene & & & & & \\
\hline
\end{tabular}

Volume 6 Issue 12, December 2017 www.ijsr.net 


\section{International Journal of Science and Research (IJSR) \\ ISSN (Online): 2319-7064}

Index Copernicus Value (2016): 79.57 | Impact Factor (2015): 6.391

\subsubsection{Image reconstruction}

Image reconstruction problems often require quite complex computations and a unique approach is needed for each application. In Ref. [8], an ADALINE network is trained to perform an electrical impedance tomography (EIT) reconstruction, i.e., a reconstruction of a $2 \mathrm{D}$ image

Applications of ANNs in these three preprocessing based on 1D measurements on the circumference of the image. Srinivasan et al. [9] trained a modiled Hopleld network to perform the inverse Radon transform (e.g., for reconstruction of computerised tomography images). The Hopleld network contained "summation" layers to avoid having to interconnect all units. Meyer and Heindl [10] used regression feed-forward networks (that learn the mapping $\mathrm{E}(\mathrm{y} \mid \mathrm{x})$, with $\mathrm{x}$ the vector of input variables and $y$ the desired output vector) to reconstruct images from electron holograms. Wang and Wahl trained a Hopleld ANN for reconstruction of 2D images from pixel data obtained from projections [11].

\subsubsection{Image restoration}

The majority of applications of ANNs in preprocessing can be found in image restoration [12-31]. In general, one wants to restore an image that is distorted by the (physical) measurement system. The system might introduce noise, motion blur, out-of-focus blur, distortion caused by low resolution, etc. Restoration can employ all information about the nature of the distortions introduced by the system, e.g., the point spread function. The restoration problem is illposed because conGicting criteria need to be fulllled: resolution versus smoothness.

The neural-network applications we reviewed had var-ious designs ranging from relatively straightforward to highly complex, modular approaches. In the most basic image restoration approach, noise is removed from an image by simple 1ltering. Greenhil and Davies [18] used a regression feed-forward network in a convolution-like way to suppress noise (with a $5 \times 5$ pixel window as input and one output node). De Ridder et al. built a modular feed-forward ANN approach that mimics the behaviour of the Kuwahara 1lter, an edge-preserving smoothing 1lter [16]. Their experiments showed that the mean squared error used in ANN training may not be representative of the problem at hand. Furthermore, un-constrained feed-forward networks often ended up in a linear approximation to the Kuwahara 11ter.

Chua and Yang [14,15] used cellular neural networks (CNNs) for image processing. A CNN is a system in which nodes are locally connected [23]. Each node con-tains a feedback template and a control template, which to a large extent determine the functionality of the network. For noise suppression, the templates implement an aver-aging function; for edge detection, a Laplacian operator. The system operates locally, but multiple iterations allow it to distribute global information throughout the nodes. Although quite fast in application, a disadvantage is that the parameters inGuencing the network behaviour (the feedback and control templates) have to be set by hand. Others have proposed methods for training CNNs, e.g., using gradient descent or genetic algorithms (grey-value images, Zamparelli [30]). CNNs were also applied for restoration of colour images by Lee and
Degyvez [21].

Another interesting ANN architecture is the gener-alised adaptive neural 1lter (GANF) [20,31] which has been used for noise suppression. A GANF consists of a set of neural operators, based on stack 1lters [12] that uses binary decompositions of grey-value data. Finally, fuzzy ANNs $[27,28]$ and the neurochips described in Ref. [22] have been applied to image restoration as well.

Traditional methods for more complex restoration problems such as deblurring and diminishing out-of-focus defects, are maximum a posteriori estimation (MAP) and regularisation. Applying these techniques entails solving high-dimensional convex optimisation tasks. The objective functions of MAP estimation or the regularisation problem can both be mapped onto the energy function of the Hop1eld network $[13,17,24,29]$. Often, mapping the problem turned out to be diOcult, so in some cases the network architecture had to be modiled as well.

Other types of networks have also been applied to im-age restoration. Qian et al. [26] developed a hybrid sys-tem consisting of order statistic 1lters for noise removal and a Hop1eld network for deblurring (by optimising a criterion function). The modulation transfer function had to be measured in advance. Guan et al. [19] developed a so-called network-of-networks for image restoration. Their system consists of loosely coupled modules, where each module is a separate ANN. Phoha and Oldham [25] proposed a layered, competitive network to reconstruct a distorted image.

\subsubsection{Image enhancement}

The goal of image enhancement is to amplify specilc (perceptual) features. Among the applications where ANNs have been developed for image enhancement [32- 42], one would expect most applications to be based on regression ANNs [37,38,40,42]. However, several enhancement approaches rely on a classiler, typically resulting in a binary output image [32,35,36,39].

The most well-known enhancement problem is edge detection. A straightforward application of regression feedforward ANNs, trained to behave like edge detectors, was reported by Pugmire et al. [38]. Chandresakaran et al. [32] used novel feed-forward architecture to classify an input window as containing an edge or not. The weights of the network were set manually instead of be-ing obtained from training. A number of more complex, modular systems were also proposed [37,40]. Formulating edge detection as an optimisation problem made it possible for Tsai et al. to train a Hopleld network for enhancement of endocardiac borders [41].

Some enhancement approaches utilise other types of ANNs. Shih et al. [39] applied an ART network for binary image enhancement. Moh and Shih [36] describe a general approach for implementation of morpho-logical image operations by a modiled feed-forward ANN using shunting mechanisms, i.e., neurons acting as switches. Waxman et al. [42] consider the applica-tion of a centre-surround shunting feed-forward ANN (proposed by Grossberg) for contrast

\section{Volume 6 Issue 12, December 2017}




\section{International Journal of Science and Research (IJSR) \\ ISSN (Online): 2319-7064}

Index Copernicus Value (2016): 79.57 | Impact Factor (2015): 6.391

enhancement and colour night vision.

\subsubsection{Applicability of neural networks in preprocessing}

There seem to be three types of problems in preprocessing (unrelated to the three possible operation types) to which ANNs can be applied:

- Optimisation of an objective function de1ned by a traditional preprocessing problem;

- Approximation of a mathematical transformation used for image reconstruction, e.g., by regression;

- Mapping by an ANN trained to perform a certain task, usually based directly on pixel data (neighbourhood input, pixel output).

To solve the 1rst type of problems, traditional methods for optimisation of some objective function may be replaced by a Hop1eld network. For a further discussion of the suitability of Hopleld networks for solving optimisation problems, see Section 3.6.

For the approximation task, regression (feed-forward) ANNs could be applied. Although some applications such ANNs were indeed successful, it would seem that these applications call for more traditional mathematical tech-niques, because a guaranteed (worst-case) performance is crucial in preprocessing.

In several other applications, regression or classilca-tion (mapping) networks were trained to perform image restoration or enhancement directly from pixel data. A remarkable 1nding was that non-adaptive ANNs (e.g., CNNs) were often used for preprocessing. Secondly, when networks were adaptive, their architectures usu-ally diIered much from those of the standard ANNs: prior knowledge about the problem was used to design the networks that were applied for image restoration or enhancement (e.g., by using shunting mechanisms to force a feed-forward ANN to make binary decisions). The interest in non-adaptive ANNs indicates that the fast, parallel operation and the ease with which ANNs can be embedded in hardware may be important criteria when choosing for a neural implementation of a specilc preprocessing operation. However, the ability to learn from data is apparently of less importance in preprocessing. While it is relatively easy to construct a linear 1lter with a certain, desired behaviour, e.g., by specifying its frequency pro1le, it is much harder to obtain a large enough data set to learn the optimal function as a high-dimensional regression problem. This holds especially when the desired network behaviour is only critical for a small subset of all possible input patterns (e.g., in edge detection). Moreover, it is not at all triv-ial to choose a suitable error measure for supervised training, as simply minimising the mean squared error might give undesirable results in an image processing setting.

An important caveat is that the network parameters are likely to become tuned to one type of image (e.g., a specilc sensor, scene setting, scale, etc.), which limits the applicability of the trained ANN. When the underlying conditional probability distributions, $\mathrm{p}(\mathrm{x} \mid \mathrm{j} \mathrm{j})$ or $\mathrm{p}(\mathrm{y} \mid \mathrm{x})$, change, the classi1cation or regres-sion network-like all statistical models - needs to be retrained.

\subsection{Data reduction and feature extraction}

Two of the most important applications of data reduction are image compression and feature extraction. In general, an image compression algorithm, used for storing and transmitting images, contains two steps: encoding and decoding. For both these steps, ANNs have been used. Feature extraction is used for subsequent segmentation or object recognition. The kind of features one wants to extract often correspond to particular geometric or perceptual characteristics in an image (edges, corners and junctions), or application dependent ones, e.g., facial features.

\subsubsection{Image compression applications}

Two diIerent types of image compression approaches can be identiled: direct pixel-based encoding=decoding by one ANN [43-51] and pixel-based encoding=decoding based on a modular approach [52-58]. DiIerent types of ANNs have been trained to perform image compression: feed-forward networks [44,49 -54,56 -58], SOMs [43,46- 48], adaptive fuzzy leader clustering (a fuzzy ART-like approach) [55], a learning vector quantiler $[49,58]$ and a radial basis function network [50]. For a more extensive overview, see [45].

Auto-associator networks have been applied to image compression where the input signal was obtained from a convolution window $[50,56,58]$. These networks contain at least one hidden layer, with less units than the input and output layers. The network is then trained to recreate the input data. Its bottle-neck architecture forces the net-work to project the original data onto a lower dimensional (possibly non-linear) manifold from which the original data should be predicted.

Other approaches rely on a SOM, which after train-ing acts as a code book $[43,46]$. The most advanced ap-proaches are based on specialised compression modules. These approaches either combine diIerent ANNs to ob-tain the best possible image compression rate or they combine more traditional statistical methods with one or more ANNs. Dony and Haykin have developed an ap-proach based on diIerent, specialised modules [53]. In their approach, a "supervisor" ANN can choose which processing module is best suited for the compression task at hand. Wang et al. also present a modular coding approach based on specialised ANNs [57].

ANN approaches have to compete with well-established compression techniques such as JPEG, which should serve as a reference. The major advantage of ANNs is that their parameters are adaptable, which may give better compression rates when trained on spe-cilc image material. However, such a specialisation becomes a drawback when novel types of images have to be compressed. For a discussion of how to evaluate image compression algorithms see, e.g., Ref. [52].

\subsubsection{Feature extraction applications}

Feature extraction can be seen as a special kind of data reduction of which the goal is to 1nd a subset of informative variables based on image data. Since image data are by nature very high dimensional, feature extrac-tion is often a necessary step for segmentation or object recognition to be successful. Besides lowering the com-putational cost, feature

\section{Volume 6 Issue 12, December 2017}

\section{www.ijsr.net}




\section{International Journal of Science and Research (IJSR) \\ ISSN (Online): 2319-7064}

Index Copernicus Value (2016): 79.57 | Impact Factor (2015): 6.391

extraction is also a means for controlling the so-called curse of dimensionality. ${ }^{1}$ When used as input for a subsequent segmentation algorithm, one wants to extract those features that preserve the class separability well $[2,3]$.

There is a wide class of ANNs that can be trained to perform mappings to a lower-dimensional space, for an extensive overview see Ref. [60]. A well-known feature-extraction ANN is Oja's neural implementation of a one-dimensional principal component analy-sis (PCA) [61], later extended to multiple dimensions [62]. In Ref. [63], Baldi and Hornik proved that training three-layer auto-associator networks corresponds to applying PCA to the input data. Later $[64,65]$, auto-associator networks with 1ve layers were shown to be able to perform non-linear dimensionality reduction (i.e., 1nding principal surfaces [66]). It is also possible to use a mixture of linear subspaces to approximate a non-linear subspace (see, e.g., Ref. [67]). Another approach to feature extraction is 1rst to cluster the high-dimensional data, e.g., by a SOM, and then use the cluster centres as prototypes for the entire cluster.

Among the ANNs that have been trained to per-form feature extraction [68-77], feed-forward ANNs have been used in most of the reviewed applications [70,74,75,77]. SOMs [7173] and Hopleld ANNs [76] have also been trained to perform feature extraction. Most of the ANNs trained for feature extraction obtain pixel data as input.

Neural-network feature extraction was performed for

- Subsequent automatic target recognition in remote sensing (accounting for orientation) [72] and character recognition [75,76];

- Subsequent segmentation of food images [74] and of magnetic resonance (mr) images [71];

- 1nding the orientation of objects (coping with rotation) $[49,70]$;

- 1nding control points of deformable models [77];

- Clustering low-level features found by the gabor 1lters in face recognition and wood defect detection [73];

- Subsequent stereo matching [69];

- Clustering the local content of an image before it is encoded [68].

In most applications, the extracted features were used for segmentation, image matching or object recognition. For (anisotropic) objects occurring at the same scale, rotation causes the largest amount of intra-class variation. ${ }^{1}$ The curse of dimensionality is a property of a classilcation or regression problem. It expresses that a higher dimensionality of the feature space leads to an increased number of parameters, which need to be estimated. The risk of over1tting the model will increase with the number of parameters, which will often lead to peaking (i.e., the best generalisation performance is obtained when using a subset of the available features) [59].

Some feature extraction approaches were designed to cope explicitly with (changes in) orientation of objects.

It is important to make a distinction between application of supervised and unsupervised ANNs for feature extraction.
For a supervised auto-associator ANN, the in-formation loss implied by the data reduction can be measured directly on the predicted output variables, which is not the case for unsupervised feature extraction by the SOM. Both supervised and unsupervised ANN feature extraction methods have advantages compared to tradi-tional techniques such as PCA. Feed-forward ANNs with several hidden layers can be trained to perform non-linear feature extraction, but lack a formal, statistical basis (see Section 5.3).

\subsection{Image segmentation}

Segmentation is the partitioning of an image into parts that are coherent according to some criterion. When considered as a classilcation task, the purpose of segmentation is to assign labels to individual pixels or voxels. Some neuralbased approaches perform segmentation directly on the pixel data, obtained either from a convolution window (occasionally from more bands as present in, e.g., remote sensing and MR images), or the information is provided to a neural classiler in the form of local features.

\subsubsection{Image segmentation based on pixel data}

Many ANN approaches have been presented that segment images directly from pixel or voxel data [78-113]. Several diIerent types of ANNs have been trained to perform pixelbased segmentation: feed-forward ANNs [90, 94, 102, 105, 106], SOMs [78,82,84,87,91,92,94,98, 102,109], Hop1eld networks [83,85,96,103,110], probabilistic ANNs [94,112], radial basis function networks [94], CNNs [108], constraint satisfaction ANNs [79] and RAM-networks [104]. A selforganising architecture with fuzziness measures was used in Ref. [86]. Also, biologically inspired neural-network approaches have been proposed: the perception model developed by Grossberg [88,89], which is able to segment images from surfaces and their shading, and the brain-like networks proposed by Opara and Worgotter [99].

Hierarchical segmentation approaches have been de-signed to combine ANNs on diIerent abstraction lev-els [105,110]. The guiding principles behind hierarchical approaches are specialisation and bottom-up process-ing: one or more ANNs are dedicated to low level fea-ture extraction=segmentation, and their results are com-bined at a higher abstraction level where another (neural) classiler performs the 1nal image segmentation. Red-dick et al. developed a pixel-based two-stage approach where a SOM is trained to segment multispectral MR images [102]. The segments are subsequently classiled into white matter, grey matter, etc., by a feed-forward ANN. Non-hierarchical, modular approaches have also been developed [78,105,107].

In general, pixel-based (often supervised) ANNs have been trained to classify the image content based on

- Texture [78,82,87,94,100,101,104,107,113];

- A combination of texture and local shape $[81,90,95$, 105,112].

ANNs have also been developed for pre- and postpro-cessing steps in relation to segmentation, e.g., for

- Delineation of contours [80,108];

\section{Volume 6 Issue 12, December 2017}




\section{International Journal of Science and Research (IJSR) \\ ISSN (Online): 2319-7064}

Index Copernicus Value (2016): 79.57 | Impact Factor (2015): 6.391

- Connecting edge pixels [103];

- Identi1cation of surfaces [88,89];

- Deciding whether a pixel occurs inside or outside a segment [110];

- Defuzzifying the segmented image [86];

and for

- Clustering of pixels $[98,109]$;

- Motion segmentation [97].

In most applications, ANNs were trained as supervised classilers to perform the desired segmentation. One feature that most pixel-based segmentation approaches lack is a structured way of coping with variations in rotation and scale. This shortcoming may deteriorate the segmentation result.

\subsubsection{Image segmentation based on features}

Several feature-based approaches apply ANNs for segmentation of images [32,71,92,114-129]. DiIerent types of ANNs have been trained to perform feature-based image segmentation: feed-forward ANNs [71,114,118, 119,125], recursive networks [127], SOMs [71,92,119 -121,129], variants of radial basis function networks [117] and CNNs [116], Hop1eld ANNs [126], principal component networks [129] and a dynamic ANN [32].

Hierarchical network architectures have been developed for optical character recognition [122] and for segmentation of range images [92].

Feature-based ANNs have been trained to segment images based on the dilerences in

- Texture [119,122-124,126-128];

- A combination of texture and local shape [118,121,125].

Besides direct classilcation, ANNs have also been used for

- Estimation of ranges [92];

- Automatic image thresholding by annealing [115] or by mapping the histogram [114];

- Estimation of the optical gow [117];

- Connecting edges and lines [116];

- Region growing [120].

- A segmentation task that is most frequently performed by feature-based anns is texture segregation, which is typically based on

- Co-occurrence matrices [118,119,128];

- Wavelet features [123];

- Multiresolution features extracted from the gabor wavelets [126];

- Spatial derivatives computed in the linear scale-space [121].

The Gabor and wavelet-based features, and features extracted from the linear scale-space provide information at several scales to the classiler, which, however, needs to cope explicitly with variations in scale. As with respect to orientation, the Gabor and wavelet-based approaches are, in general, sensitive to horizontal, vertical and diagonal features. These three directions can be combined into a local orientation measure such that rotation invariance is obtained. The scale-space features can be reduced to a few invariants that are indeed rotation invariant [130]. The generalised cooccurrence matrices cope with variations in orientation by averaging over four orthogonal orientations. Scale can also be taken into account by varying the distance parameter used to compute the co-occurrence matrix.

\subsubsection{Open issues in segmentation by ANNs}

Three central problems in image segmentation by ANNs are: how to incorporate context information, the inclusion of (global) prior knowledge, and the evaluation of segmentation approaches. In the approaches we reviewed, context information was obtained from, e.g., multiscale wavelet features or from features derived from the linear scale space (computed at a coarse scale). How context information can best be incorporated, is an interesting issue for further research. The general problem of how to include a priori knowledge in a segmentation approach is considered in Section 5.2.

A caveat is how to obtain a gold standard for the (in most cases supervised) segmentation algorithms. In gen-eral, the true class membership of the pixels=voxels in the training set is known with varying degrees of con-1dence. In Ref. [119], this problem is addressed by let-ting an expert demarcate the inner parts of areas with a similar (coherent) texture but leaving the transition areas unclassiled. Certainly, intra- and inter-observer variabil-ity needs to be assessed thoroughly (e.g., by the kappa statistic [131]) before suitable training and test images can be compiled.

Even when a reliable gold standard is available, ob-jective performance assessment entails more than simply computing error rates on novel test images. There is not yet a single measure capable of unequivocally quantify-ing segmentation quality. Besides statistical performance aspects such as coverage, bias and dispersion [131], desirable properties such as within-region homogeneity and between-region heterogeneity [132] are also impor-tant (for an overview of segmentation quality measures see Ref. [133]).

\subsection{Object Recognition}

Object recognition consists of locating the positions and possibly orientations and scales of instances of ob-jects in an image. The purpose may also be to assign a class label to a detected object. Our survey of the lit-erature on object recognition using ANNs indicates that in most applications, ANNs have been trained to locate individual objects based directly on pixel data. Another less frequently used approach is to map the contents of a window onto a feature space that is provided as input to a neural classiler.

\subsubsection{Object recognition based on pixel data}

Among the ANN approaches developed for pixel-based object recognition [39,42,67,70,72,134-179], several types of ANNs can be distinguished: feed-forward-like ANNs [70,147-149,152,164,165,171,172], variants us-ing weight sharing $[144,159,160]$, recurrent networks [179], the ART networks introduced by Grossberg [39,139], mixtures-ofexperts [173], (evolutionary) fuzzy ANNs [155], bidirectional auto-associative memories [157], the Neocognitron introduced by Fukushima [150,162] and

\section{Volume 6 Issue 12, December 2017}




\section{International Journal of Science and Research (IJSR) \\ ISSN (Online): 2319-7064}

Index Copernicus Value (2016): 79.57 | Impact Factor (2015): 6.391

variants hereof $[137,161]$, piecewise-linear neural classi1ers [168], higher-order ANNs [169,170] and Hopleld ANNs $[135,175,176]$. Besides, interesting hardware ANNs have been built for object recognition: the RAM network $[143,145]$ and optical implementations [154,167]. Finally, SOMs are occasionally used for feature extraction from pixel data $[171,177]$; the output of the map is then propagated to a (neural) classiler.

Several novel network architectures have been developed specilcally to cope with concomitant object variations in position, (in-plane or out-of-plane) rotation and scale (in one case, an approach has been developed that is invariant to changes in illumination [167]). It is clear that a distinction needs to be made between invariant recognition in 2D (projection or perspective) images and in 3D volume images. An interesting approach that performs object recognition, which is invariant to $2 \mathrm{D}$ translations, in-plane rotation and scale, is the neurally inspired what-and-where 11-ter [139]. It combines a multiscale oriented 1lter bank (what) with an invariant matching module (where). Other approaches rely on learning the variations explicitly by training [141,147,148,164]. Egmont-Petersen and Arts built a statistical intensity model of the object that should be detected [147,148]. The convolution ANN was trained using synthetic images of the (modelled) object with randomly chosen orientations. Penedo et al. developed a two-stage ANN approach for recognition of nodules in chest radiographs [164]. These ANNs were trained partly with synthetic subimages of nodules. Others have developed approaches that are invariant to both translation and $2 \mathrm{D}$ rotation $[144,178]$, or systems that through their architectures perform processing in a translation-invariant way and=or at diIerent scales (e.g., the Neocognitron [150] and the shared weight networks [158,159]). Fukumi et al. developed a hierarchical approach for rotation-invariant object recognition [70]. This approach, like its predecessor [149], maps the image to a polar space in which rotation-invariant recognition takes place.

Clearly, when object recognition is performed by teaching a classiler to recognise the whole object from a spatial pattern of pixel intensities, the complexity of the classiler grows exponentially with the size of the object and with the number of dimensions (2D versus 3D). An interesting approach that circumvents this problem is iterative search through the image for the object centre [143]. The output of the ANN is the estimated displacement vector to the object centre. Depending on the contents of the scene, even context information may be required before the objects of interest can be recognised with con1dence. The incorporation of context information may again lead to a large number of extra parameters and thereby a more complex classiler. To cope with this problem the so-called multiresolution approaches have been developed [171,175,176], which combine the intensities from pixels located on diIerent levels of a pyramid [180] but centred around the same location. This provides the classi1er with context in-formation, but a combinatorial explosion in the number of parameters is circumvented. Still, variations in scale have to be learned explicitly by the classi1er. A disad-vantage of ANN pyramid approaches is that they sample the scale space coarsely as the resolution is reduced with a factor two at each level in the pyramid (in, e.g., the linear scale space [181], scale is a continuous variable). A special type of ANN that incorporates the scale in-formation directly in a pyramidal form is the so-called higher-order ANN [169,170]. This network builds up an internal scale-space-like representation by what is called coarse coding. However, higher-order ANNs need to learn variations in scale explicitly too. They should be used with caution because the coarse coding scheme may lead to aliasing, as the high-resolution images are not blurred before computing the coarser image at the next level.

Rare conditions such as object occlusion or the occurrence of multiple objects within the (sub) image that is processed by the classiler have hardly been considered explicitly. An experimental architecture developed by McQuiod is capable of recognizing multiple objects simultaneously within an image [161] (see also Section 3.6).

Recurrent ANNs (with feed-back loops [182]) can be used to develop special approaches for object recognition [179]. The added value of a recurrent network architecture lies in its memory: the current state contains information about the past, which may constitute valuable context information. The recurrent network developed by Ziemke [179] performs a convolution with an image in order to detect oil spills. The recurrence principle introduces averaging, which can give a more robust performance.

Several of the approaches for object detection and classilcation operate on binary images [137,139,143-145]. Although binarisation simpli1es the recognition problem considerably, it generally decreases the recognition performance of an ANN.

\subsubsection{Object recognition based on features}

Several neural-network approaches have been developed for feature-based object recognition [152,164,171, 177,183-209] including: feed-forward ANNs [152,171, 177,184 187,190,193-197,200,205,207-209], Hop1eld ANNs [201], a fuzzy ANN [186] and RAM ANNs [192,202]. SOMs are occasionally used to perform feature extraction prior to object recognition [177,197], although SOMs have also been trained to perform object classi1cation [206].

The smaller variety of neural architectures devel-oped for feature-based object recognition compared to the pixel-based approaches discussed in the previous section, reGects the fact that most eIort is focused on developing and choosing the best features for the recognition task. Common for many feature-based approaches is that variations in rotation and scale are coped with by the features, e.g., statistical moments. A certain amount of noise will in Guence the computed features and deteriorate the recognition performance [203]. So the major task of the subsequent classiler is to 1lter out noise and distortions propagated by the features. Moreover, when the object to be detected is large and needs to be sampled densely, feature extraction is inevitable. Otherwise, a neural classiler will contain so many parameters that a good generalisation will be impeded.

In general, the types of features that are used for object

\section{Volume 6 Issue 12, December 2017}




\section{International Journal of Science and Research (IJSR) \\ ISSN (Online): 2319-7064}

Index Copernicus Value (2016): 79.57 | Impact Factor (2015): 6.391

recognition diler from the features used by the neural-based segmentation approaches already reviewed. For object recognition, the features typically capture local geometric properties:

- Points with a high curvature on the detected object contours [164,208];

- (gabor) 1lter banks [201,207] including wavelets [191];

- Dedicated features: stellate features [194] and ocr features [190];

- Projection of the (sub)image onto the $\mathrm{x}$ - and $\mathrm{y}$-axis [184];

- Principal components obtained from the image [204,205] (feature extraction);

- (distances to) feature space trajectories [210], which describe objects in all rotations, translations or scales [187];

- The fourier descriptors derived from the image [209];

- The zernike moments [195] and the moments of hu [203].

The Fourier descriptors, the Zernike moments and the moments of $\mathrm{Hu}$ are invariant to changes in object posi-tion, orientation and scale [203,211]. For a discussion of moments and invariance to grey-level transformations, see Ref. [211].

Multiresolution approaches have also been developed for object recognition based on features from

- The linear scale-space $[185,193]$

- The gauss pyramid [171];

- The laplace pyramid [205].

Also, the positions of detected edges (input level C) may serve as features for a classiler [171]. Finally, a set of features has been developed that is invariant to changes in colour [192].

Which set of features is best suited for a particular recognition task, depends on the variations among the objects (and of the background) with respect to position, (in-plane) orientation and scale. Knowledge of the de-grees of freedom the approach has to cope with is needed for choosing a suited set of features (feature selection is discussed in Section 5.1).

\subsubsection{Using pixels or features as input?}

Most ANNs that have been trained to perform image segmentation or object recognition obtain as input either pixel=voxel data (input level A) or a vector consisting of local, derived features (input level B). For pixel- and voxelbased approaches, all information (within a win-dow) is provided directly to the classiler. The perfect (minimal errorrate) classiler should, when based directly on pixel data, be able to produce the best result if the size of the window is comparable to that of the texture elements (texels) or the window encompasses the object and the (discriminative) surrounding background. When, on the other hand, the input to the classi1er consists of a feature vector, the image content is always compressed. Whether suOcient discriminative information is retained in the feature vector, can only be resolved experimentally.

Two-dimensional image modalities such as radiography, 2D ultrasound and remote sensing often exhibit concomitant variations in rotation and scale. If such in-variances are not built into a pixel-based ANN, careful calibration (estimation of the physical size of a pixel) and subsequent rescaling of the image to a standard resolution are required steps to ensure a con1dent result. When only rotations occur, features obtained from a polar map-ping of the window may ensure a good segmentation or detection result [70,149].

In many applications, however, calibration is unfea-sible and $2 \mathrm{D}=3 \mathrm{D}$ rotation and scale invariance needs to be incorporated into the ANN. For pixel-based ap-proaches, invariance can be either built directly into the neural classi1er (e.g., using weight sharing [159] or by taking symmetries into account [212]), or the classiler has to be trained explicitly to cope with the variation by including training images in all relevant orientations and scales. A major disadvantage of these approaches is that object variations in rotation and scale have to be learned explicitly by the classiler (translation can usually be coped with by convolution). This again calls for a very large, complete training set and a classiler that can generalise well. Model-based approaches have been presented that can generate such a complete training set $[147,148,164,185]$, see the discussion above. How to de-sign robust pixel-based algorithms for segmentation and object recognition that can cope with the three basic aOne transforms, is a challenging subject for future research.

In situations where many concomitant degrees of freedom occur (2D or 3D rotation, scale, a One grey-level transformations, changes in colour, etc.), only feature-based approaches may guarantee that the re-quired invariance is fully obtained. It is clear that when variations in orientation and scale occur and reliable calibration is unfeasible, an ANN based on invariant fea-tures should be preferred above a pixel-based approach. Another advantage of feature-based approaches is that variations in rotation and scale may remain unnoticed by the user, who may then end up with a poor result. When there is no limited set of images on which an algorithm has to work (e.g., image database retrieval), the more Gexible pixel-based methods can prove useful.

The recommendation to prefer feature-based over pixel=voxel-based image processing (when signilcant variations in rotation and scale actually occur in the image material), puts emphasis on the art of develop-ing and choosing features which, in concert, contain much discriminative power in relation to the particular image processing task. Prior knowledge regarding the image processing task (e.g., invariance) should guide the development and selection of discriminative fea-tures. Feature-based classilers will, in general, be easier to train when the chosen features cope adequately with the degrees of freedom intrinsic to the image material at hand. The removal of superGuous features is often necessary to avoid the peaking phenomenon [59] and guarantee a good generalisation ability of the classiler. This issue, which is a general problem in statistical pattern recognition, is discussed in Section 5.1.

Volume 6 Issue 12, December 2017 


\section{International Journal of Science and Research (IJSR) \\ ISSN (Online): 2319-7064}

Index Copernicus Value (2016): 79.57 | Impact Factor (2015): 6.391

\subsection{Image understanding}

Image understanding is a complicated area in image processing. It couples techniques from segmentation or object recognition with knowledge of the expected image content. In two applications, ANNs were used in combination with background knowledge to classify objects such as chromosomes from extracted structures (input level C) [213] and to classify ships, which were recog-nised from pixel data (input level A) by an advanced modular approach [214]. In another application, ANNs were used to analyse camera images for robot control from local features (input level B) [215]. Neural (decision) trees [216], semantic models based on extracted structures (input level C) [217] or neural belief networks [218] can be used to represent knowledge about the expected image content. This knowledge is then used to restrict the number of possible interpretations of single objects as well as to recognise diIerent con1gurations of image objects. Especially, the approaches by Reinus et al. [217] and Stassopoulou et al. [218] perform genuine image interpretation. Reinus trains an ANN to diagnose bone tumours. The recognition approach of Stassopoulou et al. predicts the degree of desertilcation of an area from a set of detected objects=segments, such as rocks, eroded areas, etc., in remote sensing images (input level E).

A major problem when applying ANNs for high level image understanding is their black-box character. It is virtually impossible to explain why a particular image interpretation is the most likely one. As a remedy, Stas-sopoulou et al. mapped the trained ANN onto a Bayesian belief network after training had been performed. An alternative approach to coping with the black-box problem is to use the generic explanation facility developed for ANNs [219] or to use rule extraction [220]. Another problem in image understanding relates to the level of the input data. When, e.g., seldom occurring features (input level C) or object positions (input level E) are provided as input to a neural classi1er, a large number of images are required to establish statistically representative train-ing and test sets. We feel that image understanding is the most dubious application of ANNs in the image process-ing chain.

\subsection{Optimisation}

Some image processing (sub) tasks such as graph-and stereomatching can best be formulated as optimisation problems, which may be solved by Hop1eld ANNs [11,76,103,221230]. In some applications, the Hop1eld network obtained pixel-based input (input level A) [11,76,103,226,230], in other applications the input consisted of local features (input level B) [224,228] or detected structures (typically edges, input level C) $[222,223,225,227,229]$.

Hopleld ANNs have been applied to the following optimisation problems:

- Segmentation of an image with an intensity gradient by connecting edge pixels [103,226] (input level A);

- Thresholding images by relaxation [230] (input level A);

- Two-dimensional [76,227,229] and three-dimensional object recognition by (partial) graph matching [222,228] (input level C);

- Establishing correspondence between stereo images based on features (landmarks) [224] and stereo correspondence between line cameras from detected edges [225];

- Approximation of a polygon from detected edge points [223];

- Controlling Voronoi pyramids [221].

Hopleld ANNs have mainly been applied to segmentation and recognition tasks that are too diOcult to realise with conventional neural classilers because the solutions entail partial graph matching or recognition of three-dimensional objects. Matching and recognition are both solved by letting the network converge to a stable state while minimising the energy function. It was also shown that iterating the Hopleld network can be interpreted as a form of probabilistic relaxation [231].

In most of the applications reviewed, casting the ac-tual problem to the architecture of the Hopleld network turned out to be diOcult. Occasionally, the original problem had to be modiled before it could be solved by the Hop1eld architecture. Also, convergence to a global optimum cannot be guaranteed. Finally, for Hopleld networks training and use both require complex computation, but this also holds for other more traditional algorithms for non-linear programming [232]. It should be kept in mind that some (constrained) nonlinear programming problems can be solved optimally by traditional algorithmic approaches. The Hop1eld network is really only an interesting approach for problems that lie beyond this subclass of solvable optimisation problems.

\section{Real-world applications of neural networks}

This review has concentrated on applications of ANNs to image processing problems, which were reported in the scienti1c literature. However, as the 1eld matured, ANNs have gradually found their way into a large range of (commercial) applications. Unfortunately, commercial and other considerations often impede publication of scientilc and technical aspects of such systems. In some research programmes, an overview of commercial applications of ANNs has been given, e.g., the SIENA project (ESPRIT project 9811) [233], the NeuroNet project [234] and the British NCTT project [235]. The project web sites list a number of application areas in which ANN-based systems are often encountered:

- industrial inspection: quality and process control, e.g., the detection of defect objects in the production of steel, textiles, fruit, vegetables, plants or other food products;

- document processing: computerised reading of machinegenerated and hand-written text used for,

e.g., automatic processing of forms and mail sorting;

- Identilcation and authentication: e.g., license plate recognition, 1ngerprint analysis and face detection=veri1cation [236];

- Medical diagnosis: e.g., screening for cervical cancer [237] or breast tumours [238,239];

- Defence: various navigation and guidance systems, tar-get recognition systems, etc. [240,241]. 


\section{International Journal of Science and Research (IJSR) \\ ISSN (Online): 2319-7064}

Index Copernicus Value (2016): 79.57 | Impact Factor (2015): 6.391

More information on the aforementioned applications can be found via the internet [233-235].

\section{Discussion}

Two major advantages of ANNs is that they are appli-cable to a wide variety of problems and are relatively easy to use. There are, however, still caveats and fundamental problems that need to be investigated in the future. Some of these issues are general in the sense that they are not resolved by other, competing techniques from the pat-tern recognition 1eld. Other problems are caused by the strive to solve an image processing problem by means of a statistical, dataoriented technique. Finally, some problems are fundamental to the way ANNs approach pattern recognition problems.

\subsection{Issues in pattern recognition}

When trying to solve a recognition problem, one may be faced with several problems that are fundamental to applied statistical pattern recognition: avoiding the curse of dimensionality, selecting the best features and achiev-ing a good transferability.

The 1rst problem, the curse of dimensionality, occurs when too many input variables are provided to a classi-1er or regression function. The risk of ending up with a classiler or regressor that generalises poorly on novel data, increases with the number of dimensions of the input space. The problem is caused by the inability of existing classilers to cope adequately with a large num-ber of (possibly irrelevant) parameters, a de1ciency that makes feature extraction and=or feature selection neces-sary steps in classiler development. Feature extraction has been discussed in detail in Section 3.2.2. Feature se-lection is by virtue of its dependence on a trained clas-siler, an ill-posed problem [242-244]. Besides oIering a way to control the curse of dimensionality, feature selection also provides insight into the properties of a classiler and the underlying classilcation problem [242].

A problem that is especially important in applications such as medical image processing, is how to ensure the transferability of a classiler. When trained to classify patterns obtained from one setting with a speci1c class distribution, $\mathrm{P}(! \mathrm{j})$, a classiler will have a poorer and possibly unacceptably low performance when transferred to a novel setting with another class distribution $\mathrm{P}(! \mathrm{j})$. How to cope with varying prior class distributions, is a subject for future research. Another problem related to transferability is how to account for changing underlying feature distributions, $\mathrm{p}(\mathrm{x} \mid \mathrm{l} \mathrm{j})$ or $\mathrm{p}(\mathrm{y} \mid \mathrm{x})$. In general, the parameters of the classiler or regression function need to be reestimated from a data set that is representative for the novel distribution. This problem is intrinsic to all statistical models as they are based on inductive inference. Note that for a classiler that has been trained, e.g., to recognise objects appearing at a certain scale directly from pixel data, recognition of similar objects at a diIerent scale is equivalent to classifying patterns from a novel distribution $\mathrm{p}(\mathrm{x} \mid \mathrm{!} \mathrm{j})$. Classilers or regression models that have not been retrained, should catch patterns occurring out-side the space spanned by the training cases and leave these patterns unprocessed, thereby avoiding the assign-ment of "wild-guess" class labels (see, e.g., Ref. [245]) or unreliable prediction of the conditional mean (in re-gression). Moreover, the question of how to incorporate costs of diIerent misclassilcations (again, an important topic in, e.g., medical image processing) or the computational costs of features [246], is not yet fully answered.

\subsection{Obstacles for pattern recognition in image processing}

Besides fundamental problems within the 1eld of pattern recognition, other problems arise because sta-tistical techniques are used on image data. First, most pixel-based techniques consider each pixel as a separate random variable. A related problem is how one should incorporate prior knowledge into pattern recognition techniques. Also, the evaluation of image processing approaches is not always straightforward.

A challenging problem in the application of pattern recognition techniques on images is how to incorpo-rate context information and prior knowledge about the expected image content. This can be knowledge about the typical shape of objects one wants to detect, knowl-edge of the spatial arrangement of textures or objects, or prior knowledge of a good approximate solution to an optimisation problem. According to Perlovsky [247], the key to restraining the highly Gexible learning algorithms for ANNs, lies in the very combination with prior (geometric) knowledge. However, most pattern recognition methods do not even use the prior information that neighbouring pixel=voxel values are highly correlated. This problem can be circumvented by extracting features from images 1rst, by using distance or error measures on pixel data which do take spatial coherency into ac-count (e.g., Refs. [67,248]), or by designing an ANN with spatial coherency (e.g., Ref. [159]) or contextual relations between objects (e.g., Ref. [249]) in mind. Context information can also be obtained from the pyra-mid and scale space approaches discussed in Section 3.4.1. In the reviewed applications, prior knowledge was mainly used to identify local features (input level B) that were used as input to neural classilers. Fuzzy ANNs may play a special role because they can be initialised with (fuzzy) rules elicited from domain experts. Using prior knowledge to constrain the highly parameterised (neural) classilers, is a scienti1c challenge.

There is a clear need for a thorough validation of the developed image processing algorithms. In the reviewed literature, validation on a large set of test images had only occasionally been performed. Validation and com-parison of diIerent algorithms are only possible when a reliable gold standard exists and meaningful (objective) quality measures are available. For, e.g., object recogni-tion, a gold standard is in most cases easy to obtain. In other applications, diIerent (human) observers may not fully agree about the gold standard (e.g., segmentation of medical images). Even with a reliable gold standard being available, it is clear that performance assessment entails much more than simply computing error rates on novel test images.

Finally, in image processing, classilcation and regression problems quickly involve a very large number of input dimensions, especially when the algorithms are applied

Volume 6 Issue 12, December 2017

\section{www.ijsr.net}

Licensed Under Creative Commons Attribution CC BY 


\section{International Journal of Science and Research (IJSR) \\ ISSN (Online): 2319-7064}

Index Copernicus Value (2016): 79.57 | Impact Factor (2015): 6.391

directly to pixel data. This is problematic, due to the curse of dimensionality already discussed. However, the most interesting future applications promise to de-liver even more input. Whereas, in almost all reviewed articles, ANNs were applied to two-dimensional images, e.g., (confocal) microscopy and $\mathrm{CT}=\mathrm{MR}$ (medical) imaging are threedimensional modalities. One way to cope with this increased dimensionality is by feature-based pattern recognition, another way would be to develop an architecture that inherently down samples the original im-age. As already mentioned, the search for the optimal set of features that in concert gives the best class separability is a never-ending quest. To avoid such a quest for all kinds of features that capture certain specilc aspects in a (sub)image, a general mapping (invariant to changes in position, rotation and scale) of a (sub)image to a mani-fold subspace should be developed. This will change the focus from selection of individual features to optimisa-tion of the sampling density in the invariant space.

\subsection{Neural network issues}

A number of unresolved problems exist in the 1eld of ANNs. We will in turn consider the lack of a profound theoretical basis for ANNs, the problem of choosing the best architecture and the black-box problem.

Several theoretical results regarding the approximation capabilities of ANNs have been proven. Although feedforward ANNs with two hidden layers can ap-proximate any (even discontinuous) function to an arbitrary precision, theoretical results on, e.g., the rate of convergence are lacking. For other (non)parametric classilers, the relation between the size of the train-ing set and the expected error rate has been stud-ied theoretically. One obstacle in developing a more profound statistical foundation for trained ANNs is that convergence to the global minimum of the risk function (squared error) cannot be guaranteed. Furthermore, there is always a danger of overtraining an ANN as minimising the error measure on a train-ing set does not imply 1nding a well-generalizing ANN. Nevertheless, the large body of work on application of ANNs presented in the last decade pro-vides (novice) users with many rules of thumb on how to set the various parameters. Also, methods such as regularisation, early stopping and ensemble training=bagging can help in avoiding the problem of overtraining.

Another problem is how to choose the best ANN architecture. Although there is some work on model selection [250], no general guidelines exist that guarantee the best trade-oI between bias and variance of the classiler for a particular size of the training set. Training uncon-strained networks using standard performance measures such as the mean squared error might even give very unsatisfying results. This, we assume, is the reason why in a number of applications, networks were not adaptive at all (e.g., CNNs) or heavily constrained by their architecture (e.g., the Neocognitron and shared weight networks). Note that this does not automatically imply that unconstrained ANNs should not be applied to image processing. It does indicate that as much prior knowledge as possible should be used in both ANN design and training.

ANNs suIer from what is known as the black-box problem: given any input a corresponding output is produced, but it cannot be elucidated why this decision was reached, how reliable it is, etc. In image understanding, this is certainly problematic, so the use of ANNs in such applications will remain limited. Some fuzzy neural architectures facilitate extraction of fuzzy rules after training. We expect that fuzzy ANNs will be more applicable in image understanding. In some applications, e.g., process monitoring, electronic surveillance, bio-metrics, etc., a con1dence measure is highly necessary to prevent costly false alarms. In such areas, it might even be preferable to use other, less well-performing methods that do give statistically profound con1dence intervals.

\section{Conclusion and future perspectives}

We have structured our survey according to the six steps in the image processing chain. ANNs have been trained to perform these six tasks with various degrees of success:

- Image preprocessing is a popular application area. Several (regression) ANNs were developed for image reconstruction, image restoration and image enhancement. Often, these networks were not (or only partially) adaptive. A general conclusion is that neural solutions are truly interesting when existing algorithms fail or when ANNs may reduce the amount of computation considerably. The largest risk in preprocessing is that training results in ANNs being tuned to specilc image material.

- Image compression is an interesting application of ANNs. A caveat again is tuning to particular images. As there is no unique way of evaluating compression algorithms, approaches should be compared with competing compression algorithms on novel test images. Feature extraction is a useful application of, especially, the SOM. Also, the possibility of non-linear feature extraction by feed-forward ANNs with several hidden layers oIers additional functionality.

- Image segmentation and object detection have largely been performed by pixel-based or feature-based (low level) approaches. Pixel-based approaches provide the classiler with all relevant information, but usually result in highdimensional input spaces. A feature-based approach, however, essentially compresses the information obtained from a local neighbourhood into a vector of salient features. On the one hand, it can-not be guaranteed that the chosen features comprise most of the discriminative information. On the other hand, a feature-based approach may be the only way to guarantee rotation and scale invariance. A possi-ble remedy is to develop novel pixelbased classilca-tion approaches in which neighbouring pixels are no longer regarded as completely separate variables. For object recognition, problems like object occlusion and multiple occurrences of objects remain unresolved.

- Image understanding is a dubious application of ANNs because of their black-box character and the need for a large number of images as training and test sets. As long as there is no accepted facility for explaining why a particular

\section{Volume 6 Issue 12, December 2017}




\section{International Journal of Science and Research (IJSR) \\ ISSN (Online): 2319-7064}

Index Copernicus Value (2016): 79.57 | Impact Factor (2015): 6.391

class label has been assigned to a pattern, black-box classilers will not be widely applied in im-age understanding. Neural-fuzzy architectures [251] and probabilistic networks [218] may lend themselves better for image understanding because of their trans-parent character and the possibility of initialisation by a priori rules or distributions.

- Optimisation problems have in most cases been approached by solutions based on Hop1eld ANNs.

Nevertheless, several issues remain problematic such as casting the problem at hand to the Hopleld architecture and bypassing the high dependency of the initial conlguration. Hop1eld networks become an interesting alternative to conventional optimisation techniques when the latter fail in solving the problem, either because of its non-linear character or because of the computational complexity An overview of ANN architectures used for dilerent image processing tasks is given in Table 2. It shows that feedforward ANNs, SOMs and Hopleld ANNs are the most frequently applied architectures, although many, more exotic designs have been applied to image process-ing problems as well.

\subsection{Outlook}

This article has to a large extent been an overview of what can now perhaps be called the "neural network hype" in image processing: the approximately 15-year period following the exciting publications of Kohonen [252], Hop1eld [253] and Rumelhart et al. [4]. Their work led many researchers to develop and apply various methods, which were originally inspired by the structure of the human brain. In some cases, the emphasis was on biological plausibility. Other applications focused on the possibility of parallel implementation. In most applica-tions, however, the adaptive capabilities of feed-forward ANNs were used to build a classi1er.

We believe that the last few years have seen a change in attitude towards ANNs, so that now ANNs are not anymore automatically seen as the best solution to any classilcation or regression problem. The 1eld of ANNs has to a large extent been reincorporated in the various disciplines that inspired it: pattern recognition, psychol-ogy and neurophysiology. ANNs are interesting as tools when there is a real need for an adaptive approach or a fast, parallel solution, but one should remain open to new interesting developments, such as the recently proposed support vector machines [254].

So what are the challenges left for ANNs in image processing? As we have discussed before, the main problems in many image processing applications still are the abundance of features and the diOculty of coping with concomitant variations in position, orientation and scale. This clearly indicates the need for more intelligent, in-variant feature extraction and feature selection mecha-nisms. Prior knowledge, e.g., about the aforementioned invariances or the expected image content, should play a large role in this, but could also be incorporated into the network architecture itself.
A true challenge is to use ANNs as building blocks in large, adaptive systems consisting of collaborating mod-ules. Such an adaptive system should be able to control each module and propagate feedback from the highest level (e.g., object detection) to the lowest level (e.g., pre-processing). Another interesting possibility for ANNs is what might be called onthe-job training, which makes possible the use of ANNs in changing environments. In many application areas, this would be a valuable improvement over current systems and facilitate transfer-ability between diIerent sites.

The conclusion must be that ANNs can play a role in image processing, although it might be a role as a supporting tool rather than a major one. ANNs are useful in image processing as either non-parametric classilers, non-linear regression functions, or for (un)supervised feature extraction. If, or when, the problems of ANN ap-plication outlined in this paper are gradually solved, this role may become increasingly larger.

\section{Acknowledgements}

This work was partly supported by the Dutch Cancer Foundation (KWF) on grant RUL-97-1509, the Founda-tion for Computer Science Research in the Netherlands (SION) and the Dutch Organisation for Scientilc Re-search (NWO). We are highly grateful to R.P.W. Duin, J. Kittler and L.J. van Vliet for commenting on an ear-lier, draft version of this manuscript. We also thank an anonymous referee for useful remarks and suggestions, also Mr Ravi Ranjan sir, Mr. Montosh manna Sir Ast.Proff. YIT, Jaipur and entire team of Arya Institute of Engineering and Technology and we are finally thanks to our parents.

\section{References}

[1] J. Corn1eld, Statistical classi1cation methods, Proceedings of the Second Conference on the Diagnostic Process, Computer Diagnosis and Diagnostic Methods, Chicago, 1972, pp. 108-130.

[2] P.A. Devijver, J. Kittler, Pattern Recognition: A Statistical Approach, Englewood CliIs, London, 1982.

[3] K. Fukunaga, Introduction to Statistical Pattern Recognition, 2nd Edition, Academic Press, New York, 1990.

[4] D.E. Rumelhart, G.E. Hinton, R.J. Williams, Learning internal representations by error propagation, in: D.E. Rumelhart, J.L. McClelland (Eds.), Parallel Distributed Processing: Explorations in the microstructure of Cognition, Vol. I, MIT Press, Cambridge, 1986,pp. 319362.

[5] N.R. Pal, S.K. Pal, A review on image segmentation techniques, Pattern Recognition 26 (9) (1993) $1277-$ 1294.

[6] W.R.M. Dassen, M. Egmont-Petersen, R.G.A. Mulleneers, Arti1cial neural networks in cardiology; a review, in: P.E. Vardas (Ed.), Cardiac Arrhythmias, Pacing \& Electrophysiology, Kluwer Academic Publishers, London, 1998, pp. 205-211.

[7] C.M. Bishop, Neural Networks for Pattern Recognition, Oxford University Press, Oxford, 1995. 
[8] A. Adler, R. Guardo, A neural network image reconstruction technique for electrical impedance tomography, IEEE Trans. Med. Imaging 13 (4) (1994) 594-600.

[9] V. Srinivasan, Y.K. Han, S.H. Ong, Image reconstruction by a Hopleld neural network, Image Vision Comput. 11 (1993) 278-282.

[10]R.R. Meyer, E. Heindl, Reconstruction of oI-axis electron holograms using a neural net, J. Microsc. 191 (1998) 52-59.

[11] Y.M. Wang, F.M. Wahl, Vector-entropy optimizationbased neural-network approach to image reconstruction

\section{Author Profile}

Indra Kishor received M.Tech (CSE) and B.Tech (CSE) degree from Rajasthan Technical University Kota. Raj. and also M.B.A(IT) Degree from Sikkim Manipal university with three year Diploma in Computer science from State board of Technical Education Jharkhand, and currently enhancing career in the field of Education and research.

Dhara Upadhyay working as Asst. Professor in AIET Jaipur Rajasthan and got M.Tech (CSE ) degree from GLA university, B.Tech from Hindustan University also three year Diploma from Dhaylabagh university. 